\title{
Variation of gravitational constant w. r. t. the spinning velocity of super dense stars in very strong gravitational field
}

\author{
Md Shams Nadeem ${ }^{1}$, Dipo Mahto ${ }^{2}$, Kumari Vineeta ${ }^{3}$, Krishna Murari Singh ${ }^{2}$ \\ ${ }^{1}$ Department of Physics, T. M. B. U. Bhagalpur-812007, India \\ ${ }^{2}$ Department of Physics, Marwari College, T.M.B.U. Bhagalpur-812007, India \\ ${ }^{3}$ Department of Education, S. M. College, T.M.B.U. Bhagalpur, India
}

\section{Email address:}

msn.phy@gmail.com (M. S. Nadeem),dipomahto@hotmail.com (D. Mahto), vineeta.priyadarshi@gmail.com (K. Vineeta), kmsinghphy@gmail.com (K. M. Singh)

\section{To cite this article:}

Md Shams Nadeem, Dipo Mahto, Kumari Vineeta, Krishna Murari Singh. Variation of Gravitational Constant w. r. t. the Spinning Velocity of Super Dense Stars in Very Strong Gravitational Field. American Journal of Astronomy and Astrophysics.

Vol. 2, No. 4, 2014, pp. 42-46. doi: 10.11648/j.ajaa.20140204.12

\begin{abstract}
Mahto et al. have derived the formula for the variation of the gravitational constant given by $\mathrm{G}^{\prime}=\frac{\mathrm{G}}{\sqrt{1-\mathrm{v}^{2} / \mathrm{c}^{2}}}$ in very strong gravitational field of the compact bodies like super massive black holes and neutron stars (2013). In this paper, we have extended this work to show that the variation of gravitational constant in the strong gravitational field with respect to the spinning velocity of super dense stars is directly proportional to its spinning velocity.
\end{abstract}

Keywords: Gravitational Constant, Black Hole and Neutron Star

\section{Introduction}

In 1687, Isaac Newton proposed the universal law of gravitation which states that every particle in the universe exerts a force on every particle along the line joining their centres. The magnitude of the force is directly proportional to the product of the masses of the two particles and inversely proportional to the square of the distance between them (Newton 1687 \& Mahto et al.2013).

In 1915, Albert Einstein demonstrated better theory of gravitation on the basis of general relativity, which has overcome the limitations of Newton's law of universal gravitation (Bergman1969 \& Mahto et al.2013).

In, 1951, Papapetrou first derived the equations of motion for a spinning test particle in a gravitational field. After this work, several people have considered the problem of the motion of spinning bodies in the gravitational field of a black hole or some other compact object such as a neutron star by means of post-Newtonian approximations (Ma \& Wang, 2014).

In November 2006, J. B. Fixler et al. measured the Newtonian constant of gravity, $G$, using a gravity gradiometer based on atom interferometry and reported a value of $G=6.693 \times 10^{-11} \mathrm{~m}^{3} \mathrm{~kg}^{-1} \mathrm{~s}^{-2}$, with a standard error of the mean of $\pm 0.027 \times 10^{-11}$ and a systematic error of $\pm 0.021 \times 10^{-11} \mathrm{~m}^{3} \mathrm{~kg}^{-1} \mathrm{~s}^{-2}$ (Fixler, 2007 \& Mahto et al.2013).

Mahto et al. have proposed a relation for the variation of the gravitational constant given by $G^{\prime}=G / \sqrt{1-v^{2} / c^{2}}$ in very strong gravitational field of the compact bodies applying special relativity and Newton's law of gravitation for two bodies where $\mathrm{v}$ be the velocity of spinning compact bodies like black holes, neutron stars etc.(Mahto et al. 2013).

In the present research paper, we have extended the above work to show that the variation of gravitational constant in the strong gravitational field with respect to the spinning velocity of super dense stars is directly proportional to its spinning velocity.

\section{Theoretical Discussion}

The constant of proportionality $(G)$ called gravitational constant/universal constant is one of the fundamental constants of nature. As the precision of measurements increased the disparity between the values of $G$, gathered by different groups, surprisingly increased. This unique situation was reflected by the 1998 CODATA decision to increase the relative $\mathrm{G}$ uncertainty from $0.013 \%$ to $0.15 \%$ (Mohr \& Taylor, 2001). The repetitive measurements of the 
gravitational constant $(G)$ show that $G$ varies significantly with the orientation of the test masses relative to the system of fixed stars, as was predicted by the Attractive Universe Theory. The distances between the test masses were in the decimeter range and it has been observed that the gravitational constant $(\mathrm{G})$ changes with the orientation by at least $0.054 \%$.(Mikhail et al., 2002).

Two new values of $\mathrm{G}$ resulting from two new experiments each with comparatively small uncertainties but in disagreement with each other and with earlier measurements with comparable uncertainties led to an even larger expansion of the a priori assigned uncertainties of the data for $G$ than was necessary in 2006. In both cases the expansion was necessary to reduce the inconsistencies to an acceptable level. This increase has resulted in a $20 \%$ increase in uncertainty of the 2010 recommended value compared to that of the 2006 value: 12 parts in $10^{5}$ vs. 10 parts in $10^{5}$. Furthermore, the 2010 recommended value of $G$ is smaller than the 2006 value by the fractional amount $6.6 \times$ $10^{-5}$ (Peter et al., 2012). The equation for the variability of gravitational constant in the strong gravitational field like the gravitational field of black holes, neutron stars and quasars, depending upon the spinning velocity is given by (Mahto et al.2013).

$$
G^{\prime}=\frac{G}{\sqrt{1-v^{2} / c^{2}}}
$$

The above equation can be written as

$$
\begin{gathered}
G^{\prime}=G\left(1-\frac{v^{2}}{c^{2}}\right)^{-1 / 2} \\
G^{\prime}=G\left[1+\frac{1}{2}(v / c)^{2}+\frac{3}{8}(v / c)^{4}+\frac{5}{16}(v / c)^{6}+\ldots\right]
\end{gathered}
$$

The above equation is differentiated with respect to the spinning velocity (v) of super dense stars like black holes, neutron stars etc. and solving, we have

$$
\frac{d G^{\prime}}{d v}=\frac{G v}{c^{2}}\left[1+\frac{3}{2}(v / c)^{2}+\frac{15}{8}(v / c)^{4}+\ldots\right]
$$

E.S. Reich has shown graphically in his paper that the spinning rate of the super massive black holes begin from about $50 \%$ of the speed of light to $99 \%$ of the speed of light and some super massive black holes spin at more than $90 \%$ of the speed of light, which suggest that they gained their mass through major galactic mergers (Reich, 2013 \& Mahto et al.2013). It is also clear from the graph that no super massive black holes spin at rate below than $40 \%$ of the speed of light. On the basis of data regarding the speed of super massive black holes from $10 \%$ to $98 \%$ of the speed of light (Mahto et al.2013), we can proceed the following facts as:

Since $v<c$

Or $v / c<1$

Or $(v / c)^{2}<<1$

Or $(v / c)^{3}<<<1$
Hence from the facts of the above expressions, it is clear that the terms containing in equation(4) within the big bracket except 1 will have negligible contributions and therefore, all the terms containing within the big bracket except 1 can be neglected.

$$
\frac{d G^{\prime}}{d v}=\frac{G v}{c^{2}}
$$

The terms $G$ and $c$ are the constant representing the gravitational constant and velocity of light respectively. The above equation can be written as

$$
\frac{d G^{\prime}}{d v} \alpha v
$$

The equation shows that the variation of gravitational constant in the strong gravitational field with spinning velocity of super dense stars is directly proportional to its spinning velocity.

In the case of non-spinning black holes, the spinning velocity must be zero and hence, we have

$$
v=0
$$

Putting the above in equation(3) and solving, we have

$$
G^{\prime}=G
$$

The equation (8) is applicable for all the planets for solar system and other stars having low velocities $(v / c<<1)$ than that of super dense stars like black holes. This also means that the gravitational constant does not vary at all in weak gravitational field as discussed in the Newton's laws of gravitation.

Now on the basis of equation (6), we shall calculate the variation of gravitational constant in the strong gravitational field with spinning velocity for different test super dense stars like black holes from available data.

\section{Data in Support of Variation of $G$}

There are many constants of nature in which the gravitational constant $G$ has a vital role in the study of gravitation.

Between 1973 and 2010, the lowest average value of G was 6.6659 , and the highest 6.734 , a 1.1 percent difference. These published values are given to at least 3 places of decimals, and sometimes to 5 , with estimated errors of a few parts per million. Either this appearance of precision is illusory, or $\mathrm{G}$ really does change. The difference between recent high and low values is more than 40 times greater than the estimated errors. This is expressed as standard deviations. The real change in the gravitational constant (G) will affecte the changes in the earth's astronomical environment, as the earth moves around the sun and as the solar system moves within the galaxy or may be there are inherent fluctuations in the gravitational $(\mathrm{G})$. Such changes would never be noticed as long as measurements are averaged over time and averaged across laboratories (Rupert Sheldrake).

From a least squares fit of the parameters of the solar system model to the data taken from various range measurements including those by Viking launders to Mars 
from July 1976 to July 1982, a bound on $\dot{G}$ is obtained: $\dot{G} / G=(2 \pm 4) \times 10^{-12} y r^{-1}$ (Hellings et al. 1983).

From the analysis of the data from 1969 to 1990, a bound on $\dot{G}$ is obtained: $\dot{G} / G=(0.1 \pm 10.4) \times 10^{-12} y r^{-1}$, while from the data from 1970 to $1994, \dot{G} / G=(1 \pm 8) \times 10^{-12} y r^{-1}$. Recent analysis using the data up April 2004 yields $\dot{G} / G=(4 \pm 9) \times 10^{-13} y r^{-1}$. The uncertainty for $\dot{G} / G$ is improving rapidly since the sensitivity for the observations depends on the square of the time span (Chiba 2011 \& Mahto et al.2013).

From the timing of the binary pulsar PSR 1913+16, a bound on $\dot{G}$ is obtained: $\dot{G} / G=(1.0 \pm 2.3) \times 10^{-11} y r^{-1}$ (Damour et al. 1988 \& Mahto et al.2013).
Jin Wang studied the astrophysical bounds on the change of the gravitational constant with time and found that $\dot{G} / G<10^{-12} y r^{-1}$ is the condition that has to be satisfied in order not to cause a conflict with the observations (Wang, 1991).

\section{Data Used}

1. $\mathrm{G}=6.67384 \times 10^{-11} \mathrm{~m}^{3} \mathrm{Kg}^{-1} \mathrm{~s}^{-2}$.

2. Speed of super dense $\operatorname{stars}(\mathrm{v})=10 \%$ to $98 \%$ of speed of light (c).

3. Speed of light $(\mathrm{c})=3 \times 10^{8} \mathrm{~m} / \mathrm{s}$.

4. Speed (v) of Super massive black holes, Neutron stars, Quasars $(\mathrm{m} / \mathrm{s})=0.9 \times 10^{7}$ to $2.94 \times 10^{8} \mathrm{~m} / \mathrm{s}$

\section{Table}

Variation of gravitational constant w.r.t. the spinning velocity of super dense stars in very strong gravitational field

\begin{tabular}{|c|c|c|c|}
\hline SI. No & $\begin{array}{l}\% \text { speed of super massive black holes, } \\
\text { Neutron stars, Quasars of the speed of light }\end{array}$ & $\begin{array}{l}\text { Speed (v) of Super massive black holes, } \\
\text { Neutron stars, Quasars (m/s) }\end{array}$ & $\left(\mathrm{m}^{2} / \mathrm{kgs}\right) . \quad \frac{d G^{\prime}}{d v}=\frac{G v}{c^{2}}$ \\
\hline 1 & 3 & $0.9 \times 10^{7}$ & $.666 \times 10^{-20}$ \\
\hline 2 & 4 & $1.2 \times 10^{7}$ & $.888 \times 10^{-20}$ \\
\hline 3 & 5 & $1.5 \times 10^{7}$ & $1.11 \times 10^{-20}$ \\
\hline 4 & 10 & $3.0 \times 10^{7}$ & $2.22 \times 10^{-20}$ \\
\hline 5 & 15 & $4.5 \times 10^{7}$ & $3.33 \times 10^{-20}$ \\
\hline 6 & 20 & $6.0 \times 10^{7}$ & $4.44 \times 10^{-20}$ \\
\hline 7 & 25 & $7.5 \times 10^{7}$ & $5.55 \times 10^{-20}$ \\
\hline 8 & 30 & $9.0 \times 10^{7}$ & $6.66 \times 10^{-20}$ \\
\hline 9 & 35 & $1.05 \times 10^{8}$ & $7.77 \times 10^{-20}$ \\
\hline 10 & 40 & $1.20 \times 10^{8}$ & $8.88 \times 10^{-20}$ \\
\hline 11 & 45 & $1.35 \times 10^{8}$ & $9.99 \times 10^{-20}$ \\
\hline 12 & 50 & $1.50 \times 10^{8}$ & $11.10 \times 10^{-20}$ \\
\hline 13 & 55 & $1.65 \times 10^{8}$ & $12.10 \times 10^{-20}$ \\
\hline 14 & 60 & $1.80 \times 10^{8}$ & $13.32 \times 10^{-20}$ \\
\hline 15 & 65 & $1.95 \times 10^{8}$ & $14.43 \times 10^{-20}$ \\
\hline 16 & 70 & $2.10 \times 10^{8}$ & $15.54 \times 10^{-20}$ \\
\hline 17 & 75 & $2.25 \times 10^{8}$ & $16.65 \times 10^{-20}$ \\
\hline 18 & 80 & $2.40 \times 10^{8}$ & $17.76 \times 10^{-20}$ \\
\hline 19 & 85 & $2.55 \times 10^{8}$ & $18.87 \times 10^{-20}$ \\
\hline 20 & 90 & $2.70 \times 10^{8}$ & $19.98 \times 10^{-20}$ \\
\hline 21 & 95 & $2.85 \times 10^{8}$ & $21.09 \times 10^{-20}$ \\
\hline 22 & 98 & $2.94 \times 10^{8}$ & $21.75 \times 10^{-20}$ \\
\hline
\end{tabular}




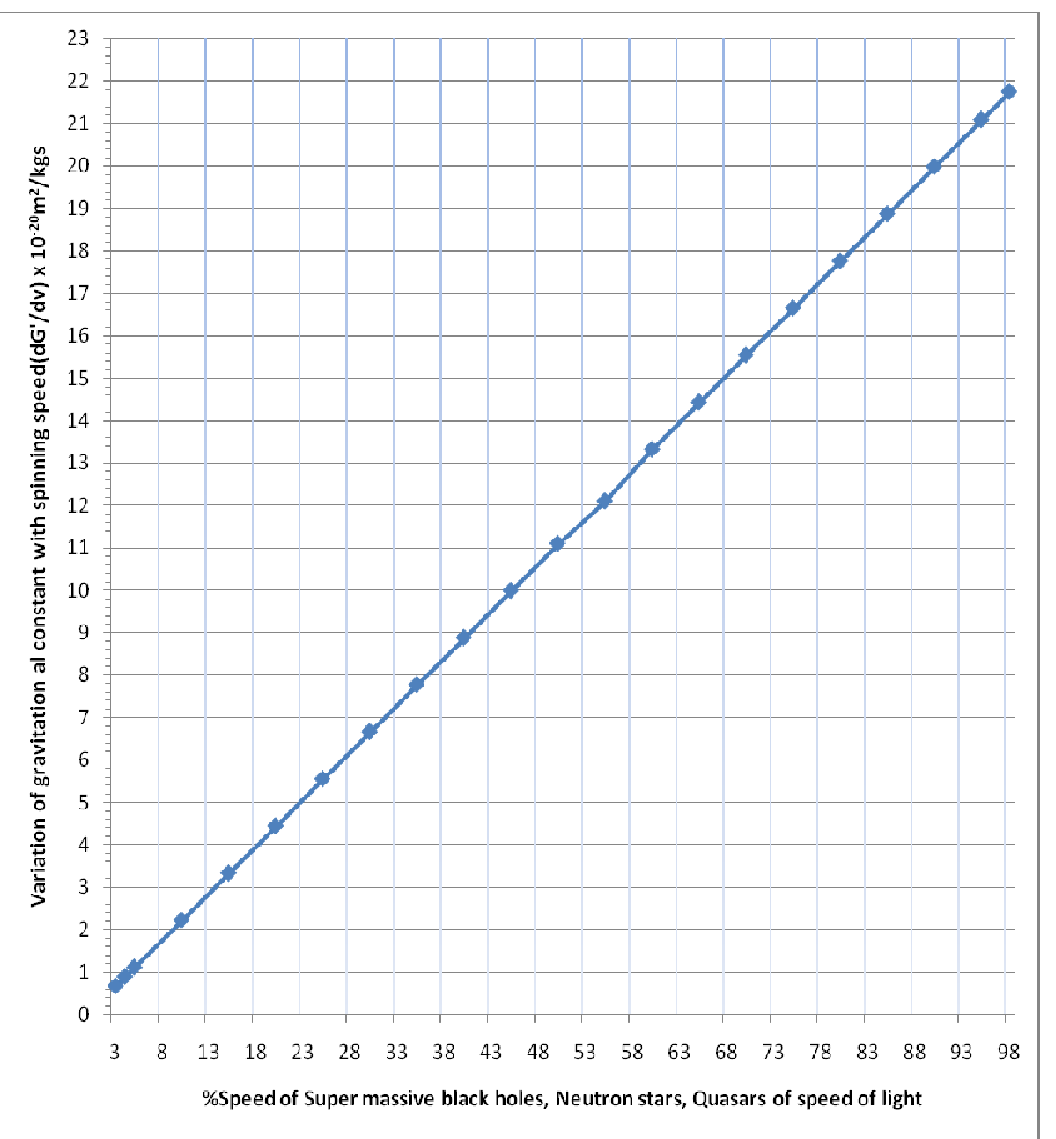

Figure 1. The graph plotted between variation of gravitational constant w.r.t. velocity $\left(d G^{\prime} / d v\right)$ and \% speed of super massive black holes, neutron stars and quasars of speed of light.

\section{Results and Discussion}

Mahto et al. have derived the formula for the variation of the gravitational constant given by $G^{\prime}=G / \sqrt{1-v^{2} / c^{2}}$ in very strong gravitational field of the compact bodies like black holes and neutron stars applying special relativity and Newton's law of gravitation for two bodies where $v$ be the velocity of spinning compact bodies like black holes, neutron stars etc. and $\mathrm{c}$ be the velocity of light (2013). In the present work, we have extended this work to derive an expression for the variation of the gravitational constant $(\mathrm{G})$ with respect to the spinning velocity which shows that the variation of gravitational constant in the strong gravitational field with respect to the spinning velocity of super dense stars is directly proportional to its spinning velocity. We also have calculated their values for different super dense stars at different speed of the spinning of the black holes, neutron stars and quasars.

To know the nature of variation of the gravitational constant $(\mathrm{G})$ with respect to the spinning velocity which shows that the variation of gravitational constant in the strong gravitational field with respect to the spinning velocity of super dense stars, the graph is plotted between the speed of the black holes, neutron stars and quasars and corresponding variation of gravitational constant in the strong gravitational field with respect to the spinning velocity $\left(\mathrm{dG}^{\prime} / \mathrm{dv}\right)$, the straight line nature of the graph is obtained. This shows the unifom variation of $\mathrm{dG}^{\prime} / \mathrm{dv}$ with the spinning velocity of super dense stars.

It has been observed from the table and graph that the variability of the gravitational constant $\left(\mathrm{dG}^{\prime} / \mathrm{dv}\right)$ increases linearly with the increase of the speed of the black holes, neutron stars and quasars.

\section{Conclusion}

In course of the present research work, we can draw the following conclusions such as:

(1) The variation of the gravitational constant with spinning velocity $\left(d G^{1} / d v\right)$ increases linearly with the increase of the spinning rate of super dense stars like the black holes, neutron stars and quasars.

(2) The linear relationship between the variation of the gravitational constant with spinning velocity and velocity gives the validity of the formula of $G^{\prime}=G / \sqrt{1-v^{2} / c^{2}}$, because the present relation has been derived from this formula.

(3) These variations are only possible in very strong gravitational field with high spinning rate of super dense stars.

(4) The variation of gravitational constant in the strong gravitational field with spinning velocity of super dense stars is directly proportional to its spinning velocity. 


\section{Acknowledgement}

Authors are also grateful to the referee for pointing out the errors in the original manuscript and making constructive suggestions and we are also obliged to Dr. M.S.H. John, principal Marwari College Bhagalpur for his inspirations.

\section{References}

[1] I. Newton,: "The Principia (The mathematical principles of Natural knowledge)", 1667.

[2] P.G. Bergmann,: "Introduction to the Theory of Relativity". Prentice Hall of India, New Delhi (1969).

[3] T. Ma, and S. Wang,: Gravitational field equation and theory of dark matter and dark energy, Discrete and continuous dynamical system, Volume 34, Number 2, February 2014

[4] J. B. Fixler; G. T. Foster; J. M. McGuirk; M. A. Kasevich: Atom Interferometer Measurement of the Newtonian Constant of Gravity Science 315 (5808): 74-77, 2007.

[5] D. Mahto, M. S. Nadeem, U. Prasad, K. Vineeta: Study of variation of gravitational constant $(G)$ in very strong gravitational field, International Journal of Journal of Astrophysics and Space science, 2013.

[6] E. S. Reich: "Spin rate of black holes pinned down." Nature, Vol.500,p-135, Macmillan Publishing limited, August, 2013.
[7] J. Wang: "A strophysical constraints on the gravitational constant", Astrophysics and Space Science, 184, 31-36(1991).

[8] R.W. Hellings, P.J. Adams, J.D. Anderson, M.S. Keesey, E.L. Lau, E.M. Standish, V.M. Canuto, and I. Goldman: Experimental test of the variability of $\mathrm{G}$ using Viking Lander ranging data. Phys. Rev. Lett, 51, 1609-1612, 1983.

[9] A. T. Chiba: "The Constancy of the Constants of Nature: Updates." Progress of Theoretical Physics, Vol.126, No. 6, Dec. 2011.

[10] T. Damour, G.W. Gibbons and J. H. Taylor, Physical Review Letter,61, (1988), 1151.

[11] P. J. Mohr \& B.N.Taylor: The fundamental physical constants. Phys. Today 6, 2001.

[12] L. Mikhail, Gershteyn, Lev I. Gershteyn, Arkady Gershteyn, Oleg V. Karagioz: Experimental evidence that the gravitational constant varies with orientation. Gravitation \& Cosmology, volume 8, issue 3, 2002.

[13] J. Peter, Mohr, N. Barry, B. David \& Newell: CODATA Recommended Values of the Fundamental Physical Constants: 2010, National Institute of Standards and Technology, Gaithersburg, Maryland 20899-8420, USA, March 27, 2012. arXiv:1203.5425v1[Physics.atom-ph] 24 Mar 2012.

[14] www.sheldrake.org/.../how-the-universal-gravitational-const ant-varies. 